\title{
Influence of a repump laser on a nearly degenerate four-wave-mixing spectrum in atomic vapors
}

\author{
Wei Jiang* Qun-feng Chen, Yong-sheng Zhang, and G. -C. Guo \\ Key Laboratory of Quantum Information, \\ University of Science and Technology of China, \\ Hefei, 230026,P. R. China
}

(Dated: December 26, 2018)

\begin{abstract}
The influence of a repump laser on a nearly degenerate four-wave-mixing (NDFWM) spectrum was investigated. We found the amplitude and line shape of the NDFWM depended strongly on the detuning of the repump field. A five-peak structure was observed. And at some certain repump detuning a dip appeared at the central peak. A rough analysis was proposed to explain this effect.
\end{abstract}

PACS numbers: 42.65.Hw, 32.80.-t

\section{INTRODUCTINON}

Nearly degenerate four-wave mixing (NDFWM) has been extensively studied during last three decades, because it has many applications, such as phase conjugating mirror and NDFWM spectroscopy [1, 2, 3, 4, 5, 6, 7, 8]. In the latter case people are interested in the line shape of the spectrum. This spectrum can provide important information about the relaxation of states due to reservoir coupling. Many experiments employed alkali vapor as the nonlinear medium. Various theoretical models were presented 9, 10, 11]. These models were based on a two-level system (TLS) driven by a pump field. However in the real case a pure TLS is hard to find, one must consider the influence of Zeeman degeneracy and other effects. In this paper we report an experimental study of NDFWM when a repump field is applied. We show that this repump field not only increases the NDFWM signal dramatically but also affects the lineshape of the NDFWM spectrum in a profound way. We present a simple model to explain one single phenomenon. More efforts are still needed to fully understand the phenomena that we observed.

\section{EXPERIMENT}

We used the D1 transition of ${ }^{85} R b$ to produce the NDFWM signal and a repump laser was tuned to D2 line of ${ }^{85} R b$. Fig. 1 shows the energy diagram of the atom and schematic setup of the experiment. The pump field was detuned from $5 S_{1 / 2}(F=2) \rightarrow 5 P_{1 / 2}(F=3)$ transition. $\Delta$ is detuning of pump laser from this transition. The probe laser scanned across this transition. The relative detuning of probe field to pump field is $\delta$. The repump laser was tuned near $5 S_{1 / 2}(F=3) \rightarrow 5 P_{3 / 2}$ manifold with detuning $\Delta_{r} . \Delta_{r}$ is the detuning from $5 S_{1 / 2}(F=3) \rightarrow 5 P_{3 / 2}(F=2)$ transition. All these

*Electronic address: jwayne@mail.ustc.edu.cn lasers are external cavity diode laser (ECDL) and have a linewidth below $1 \mathrm{MHz}$. The powers of pump and probe fields are both $0.6 \mathrm{~mW}$. The power of repump field is $3 \mathrm{~mW}$. In order to minimize the influence of Doppler broadening, the angle between probe and pump beam were kept small (about $4 \mathrm{mrad}$ ). Diameters of the probe and pump beam are about $1 \mathrm{~mm}$. After passing the vapor cell the forward pump beam was reflected back to form the backward pump beam and overlapped with the forward pump beam. The pump and probe laser were both linearly polarized. The generated NDFWM signal was pick up by a $50 / 50$ beam splitter and directed to a photodiode detector. The temperature of the vapor cell was about $60^{\circ} \mathrm{C}$, which resulted in an atomic density $3.5 \times 10^{11} / \mathrm{cm}^{3}$.

Fig. 2 shows the recorded signal without repump beam. The signal has a triplet structure. This phenomenon has been reported in Ref. [8], but was not properly explained. In Ref. 8 the three peaks were said to be located at $\delta=0$ and $\delta= \pm \Omega^{\prime}$, where $\Omega^{\prime}=\sqrt{\Delta^{2}+\Omega^{2}}$ is the generalized Rabi frequency and $\Omega$ is Rabi frequency of the forward pump field. And a picture based on dressedstate was given. However this is not correct. Actually the two side peaks were located at $(\delta= \pm 2 \Delta)$ respectively [10]. Fig. 3 are positions of NDFWM peaks versus pump detuning $\Delta$. We can see the experimental data and the theoretical curve are in good agreement. Note that the produced NDFWM signal is rather small. This is because of the optical pumping effect of the pump and probe fields. These fields pumped most of the atoms to $5 S_{1 / 2}(F=3)$ manifold through optical pumping process. Atoms in this internal state will not contribute to the NDFWM process, thus leads to a small signal. However when we added a repump laser to pump these atoms back to the $5 S_{1 / 2}(F=2)$ manifold, the produced NDFWM signal was dramatically changed. First the signal increased significantly (Increased by a factor 20 when repump had an appropriate detuning). This is easy to understand because the atoms which can produce the NDFWM signal was increased due to hyperfine optical pumping of the repump laser. It is more interesting that the lineshape of the NDFWM signal changed dramat- 
ically too. We found this change depended sensitively on the detuning of the repump laser. Fig. 4 shows the observed NDFWM signal with a fixed pump detuning $\Delta \approx 105 \mathrm{MHz}$ and various repump detuning. The most remarkable change was that the original triplet structure changed to a five-peak structure $\left(\Delta_{r}=-60 \mathrm{MHz}\right)$. And at certain repump detuning a dip appeared at the central peak. The relative amplitude of these peaks also depended sensitively on the detuning of the repump laser. The dip at the central peak appeared when the detuning of repump field is about $-79 \mathrm{MHz}$. The FWHM of the dip was sub-Doppler and was dominated by the residual Doppler width due to imperfect probe-pump aligning. As pointed out in an early work by Berman [11], narrow structure was expected to appear whenever the system did not conserve population, orientation or alignment.

\section{DISCUSSIONS}

The five-peak structure is hard to explain although interesting. Here we only give a possible explanation of these phenomena. The change of amplitudes of these peaks maybe owing to the hole burning effect and the energy level degeneracy. Because the linewidth of the repump laser is narrow, it will burn a hole on the Maxwell velocity distribution of the atoms. That is atoms with a certain velocity will experience the hyperfine optical pumping effect. When the repump laser is scanned, atoms with different velocity will be pumped back to $5 S_{1 / 2}(F=2)$ manifold. Consequently this will cause different lineshape of NDFWM signal at different repump detuning $\Delta_{r}$. In the following we will try to explain one single phenomenon that we observed. That is the dip in Fig. 4(c).

Consider a two level system as showed in Fig. 5. $\gamma_{1}$, $\gamma_{2}$ are total relaxation rates of level 1 and 2 respectively. $\gamma_{2 \rightarrow 1}$ are spontaneous decay from level 2 to level 1. $\Delta=$ $\omega-\omega_{0}$ and $\delta=\omega_{p}-\omega$ are detunings of the pump field and the probe field respectively. We adopt the treatment in Ref. [10]. The equation which governs the evolution of density matrix $\rho$ is given by,

$$
i \hbar\left(\frac{\partial}{\partial t}+\vec{v} \cdot \nabla\right) \rho=\left[H_{0}, \rho\right]+[V, \rho]-\frac{i \hbar}{2}[\Gamma, \rho]+\left.i \hbar \frac{d \rho}{d t}\right|_{s p}+\left.i \hbar \frac{d \rho}{d t}\right|_{p h}+i \hbar \Lambda,
$$

where $H_{0}$ is free Hamiltonian of the system, $V$ is the interaction term, $\vec{v} \cdot \nabla$ accounts for the motion of the atoms. $\Gamma$ represents decay to the reservoir. $\left.\frac{d \rho}{d t}\right|_{s p}$ and $\left.\frac{d \rho}{d t}\right|_{s p}$ describe decay from 2 to 1 and decay of coherence between them respectively. $\Lambda$ is the term to account for incoherent pumping. The equations for the matrix elements are,

$$
\begin{aligned}
& i \hbar\left(\frac{\partial}{\partial t}+\vec{v} \cdot \nabla\right) \rho_{11}=\left(V_{12} \rho_{21}-c . c .\right)-i \hbar \gamma_{1} \rho_{11}+i \hbar \gamma_{2 \rightarrow 1} \rho_{22}+i \hbar \lambda_{1}, \\
& i \hbar\left(\frac{\partial}{\partial t}+\vec{v} \cdot \nabla\right) \rho_{22}=-\left(V_{12} \rho_{21}-c . c .\right)-i \hbar \gamma_{2} \rho_{22}+i \hbar \lambda_{2}, \\
& i \hbar\left(\frac{\partial}{\partial t}+\vec{v} \cdot \nabla\right) \rho_{12}=-\hbar \omega_{0} \rho_{12}+\left(V_{12} \rho_{22}-\rho_{11} V_{21}\right)-i \hbar \gamma_{p h}^{T} \rho_{12},
\end{aligned}
$$

where $\gamma_{p h}^{T}=\frac{1}{2}\left(\gamma_{1}+\gamma_{2}\right)+\gamma_{p h}$.

The third order nonlinear polarization generated by $E_{f}, E_{b}$ and $E_{p}$ is $P^{(3)}=\chi^{(3)} E_{f} E_{b} E_{p}^{*}$. The phase matching conditions result in the signal field, $E_{s}$, counterpropagating with the probe beam. If the pump fields are at frequency $\omega$ and the probe field at frequency $\omega+\delta$, then by energy conservation the frequency of the signal is $\omega-\delta$. Solving the density equations (24) in a perturbation manner can yield,

$$
\begin{aligned}
P^{(3)}= & -\frac{N_{0} \mu_{12}}{4} \Omega_{f} \Omega_{b} \Omega_{p}^{*} e^{-i\left[(\omega-\delta) t+\vec{k}_{p} \cdot \vec{r}\right]} \frac{1}{-(\Delta-\delta)-\vec{k}_{p} \cdot \vec{v}+i \gamma_{p h}^{T}} \\
& \times\left[\frac{1-R}{\delta-\Delta \vec{k} \cdot \vec{v}+i \gamma_{1}}+\frac{1+R}{\delta-\Delta \vec{k} \cdot \vec{v}+i \gamma_{2}}\right]\left[\frac{1}{-\Delta+\vec{k}_{f} \cdot \vec{v}+i \gamma_{12}}+\frac{1}{(\delta+\Delta)-\vec{k}_{p} \cdot \vec{v}+\gamma_{12}}\right]+c . c,
\end{aligned}
$$

where $\Omega_{i}(i=f, b, p)$ is the Rabi frequency $\mu_{12} E_{i} / \hbar$ associated with optical field $E_{i}$. $N_{0}$ is the equilibrium pop- ulation difference,

$$
\left(\rho_{11}-\rho_{22}\right)_{e q}=\frac{\lambda_{1}}{\gamma_{1}}-\frac{\lambda_{2}}{\gamma_{2}}\left(1-\frac{\gamma_{2 \rightarrow 1}}{\gamma_{1}}\right)
$$

The spectrum lineshape is determined by the decay 
parameters mentioned above. Two situations are of special interest in our case. Fig. 6(a) and (b) shows the two NDFWM spectrum without integration over velocity distribution. In Fig. 6(a), where $\gamma_{1}<\gamma_{2}-\gamma_{2 \rightarrow 1}$, we can see the triplet structure. A narrow peak in center and two broader peaks at the wings. The linewidth of the center peak is determined by $\gamma_{1}$. When $\gamma_{1}>\gamma_{2}-\gamma_{2 \rightarrow 1}$ the spectrum (Fig. 6(b)) is quite different with the one shown in Fig. 6(a). While the essential difference is that a dip appeared in the center of the spectrum. Fig. 6(c) and (d) are the spectrums after integration over velocity. We can see the dip still exists in Fig. 6(d), but the two side peaks are washed out by the integration.

Compare the phenomena we observed with the theory we can see that when a repump laser with appropriate detuning is added the effective decay rate $\gamma_{2}-\gamma_{2 \rightarrow 1}$ is dramatically reduced. Consequently a dip will appear at the center of the spectrum. However quantitatively comparison between experimental data and theoretical values is impossible because the model we use is overly simplified. One must take the level degeneracy into account. And the hole burning effect and power broadening of the repump laser should also be taken into consideration. Therefore careful and complex calculation is needed to achieve this goal.

We also changed the polarizations of pump, probe and repump beam respectively. We found that the NDFWM signal was insensitive to these changes. Only small variation of signal amplitude was observed.

In order to study the influence of the repump power on the NDFWM signal, we did the experiment with different repump powers. We found when we increased the repump power the signal increased while showed some saturation. When the repump power was high enough, the dip in the center of the spectrum disappeared. This is because the power broadening caused by the repump laser washed out this tiny structure.

Finally we want to mention that we noticed there was a similar work reported by Zhu et. al. 12. But the phenomenon they observed was totally different. In their work the D2 transitions of ${ }^{85} R b$ were used to produce NDFWM signal and a repump laser was tuned to the D1 transitions. They found no significant changes except the signal was amplified several times. We conjecture that this is because their lasers were so strong that saturation and power broadening became the dominant effects.

\section{CONCLUSION}

In conclusion, we have studied the influence of a repump laser on a NDFWM spectrum. We found the amplitude and line shape of the NDFWM depended strongly on the detuning of the repump field. A five-peak structure was observed. And at a certain repump detuning a dip appeared at the central peak. A rough analysis was proposed to explain this effect. More efforts are still needed to fully understand the phenomena that we observed.

\section{Acknowledgments}

This work was funded by National Fundamental Research Program (2001CB309300), National Natural Science Foundation of China (Grant No. 60121503, 10304017), the Innovation funds from Chinese Academy of Sciences.
[1] Optical Phase Conjugation, edited by R. Fisher (Acadamic Press, New York, 1983).

[2] P. F. Liao, D. M. Bloom, and N. P. Economou, App. Phys. Lett. 32, 813 (1978).

[3] D. Bloch, R. K. Raj, K. S. Peng, and M. Ducloy, Phys. Rev. Lett. 49, 719 (1982).

[4] G. Grynberg, M. Pinard, and P. Verkerk, Opt. Commun. 50, 261 (1984).

[5] M. Oria, D. Bloch, M. Fichet, and M. Ducloy, Opt. Lett. 4, 1082 (1989).

[6] Jing Liu, D. G. Steel, Phys. Rev. A 38, 4639 (1988).

[7] A. Lezama, G. C. Cardoso, and J. W. Tabosa, Phys. Rev. A 63, 013805 (2000).

[8] Jun Lin, A. I. Rubiera, and Y. F. Zhu, Phys. Rev. A 52, 4882 (1995).

[9] R. W. Boyd, M. G. Raymer, P. Narum and D. J. Harter, Phys. Rev. A 24, 411 (1981).

[10] D. G. Steel, J. T. Remillard, Phys. Rev. A 36, 4330 (1987).

[11] P. R. Berman, D. G. Steel, G. Khitrova and J. Liu, Phys. Rev. A 38, 252 (1988).
[12] Y. F. Zhu, T. N. Wasserlauf, and P. Sanchez, Phys. Rev. A 55, 668 (1997).

\section{Figure Captions}

Fig. 1 Experimental Setup. P, polarizer; BS, 50/50 beam splitter; $\mathrm{D}$, photo diode detector. The angle between probe and pump beam were about $4 \mathrm{mrad}$. Diameters of the probe and the pump beam were about $1 \mathrm{~mm}$. After passing the vapor cell the forward pump beam was reflected back to form the backward pump beam and overlapped with the forward pump beam. The pump and probe beams were $795 \mathrm{~nm}$ lasers tuned near the $5 S_{1 / 2}(F=2) \rightarrow 5 P_{1 / 2}(F=3)$ transition of ${ }^{85} R b$ with detuning $\Delta$ and $\delta$ respectively. The repump beam was a $780 \mathrm{~nm}$ laser tuned near $5 S_{1 / 2}(F=3) \rightarrow 5 P_{3 / 2}$ manifold with detuning $\Delta_{r}$. $\Delta_{r}$ is the detuning from $5 S_{1 / 2}(F=3) \rightarrow 5 P_{3 / 2}(F=2)$ transition. 
Fig. 2 NDFWM spectrum without repump beam. The pump detuning $\Delta=115 \mathrm{MHz}$. $\delta$ is the relative detuning from the frequency of central peak.

Fig. 3 Peak positions of three NDFWM resonance versus pump detuning $\Delta$.

Fig. 4 NDFWM spectrum with repump field turned on and various repump detuning $\Delta_{r}$. From (a) to (j) the repump detuning $\Delta_{r}$ are $-205 \mathrm{MHz},-132 \mathrm{MHz},-79 \mathrm{MHz}$, $-60 \mathrm{MHz},-15 \mathrm{MHz}, 93 \mathrm{MHz}, 122 \mathrm{MHz}, 163 \mathrm{MHz}, 168 \mathrm{MHz}$ and $317 \mathrm{MHz}$ respectively.
Fig. 5 Simple two-level system. $\gamma_{1}$ and $\gamma_{2}$ are total decay rate of level 1 and 2 respectively. $\gamma_{2 \rightarrow 1}$ is the decay rate from 2 to 1 .

Fig. 6 Calculated NDFWM spectrum based on Eq.(5) with different parameters. (a) $\Delta=50, \gamma_{1}=3, \gamma_{2}=$ $6, \gamma_{p h}=3, \gamma_{2 \rightarrow 1}=6$. (b) $\Delta=50, \gamma_{1}=3, \gamma_{2}=$ $0.1, \gamma_{p h}=3, \gamma_{2 \rightarrow 1}=6$. (c) the same as (a) but the spectrum was integrated over velocity distribution. (d) the same as (b) but the spectrum was integrated over velocity distribution. 

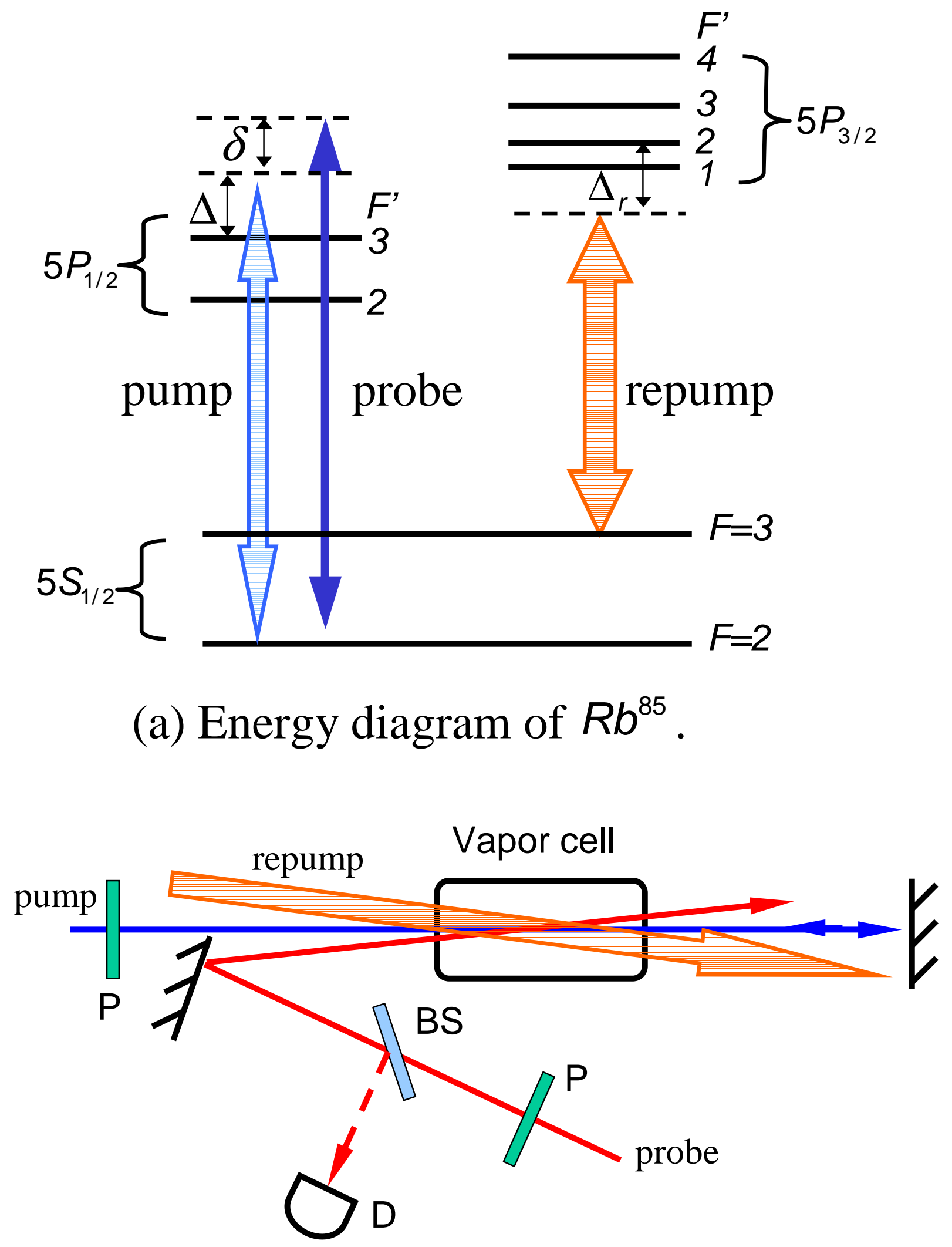

(b) Experimental setup 



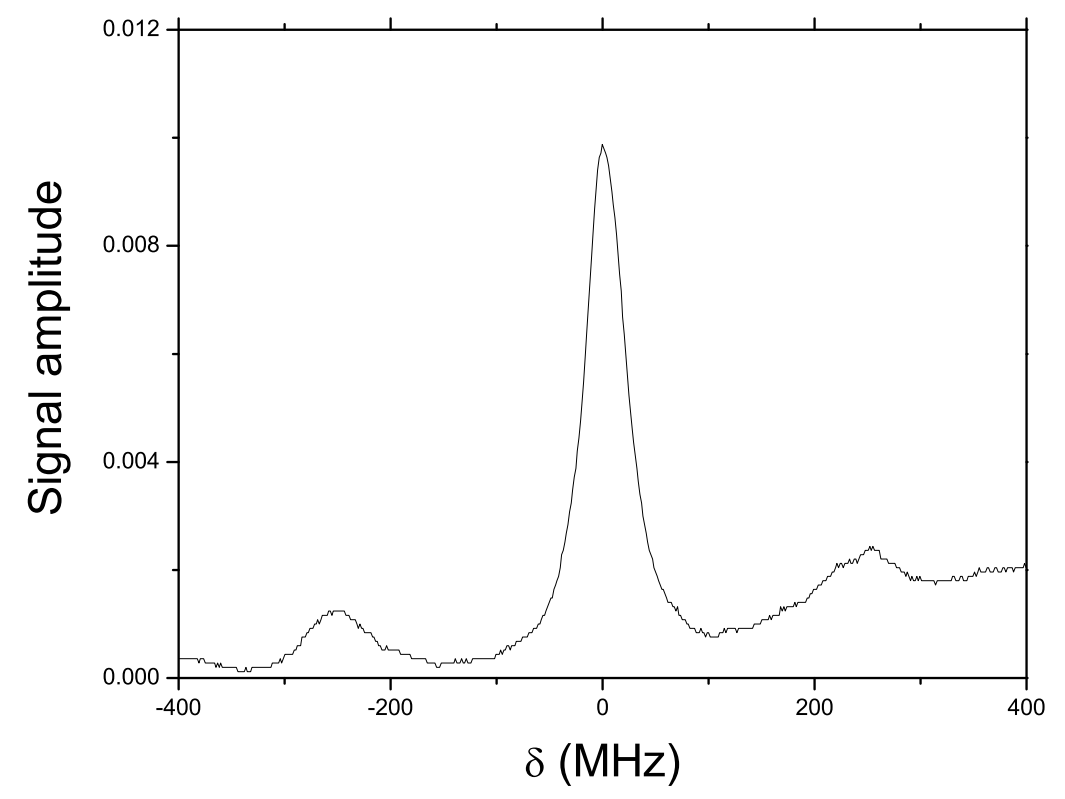




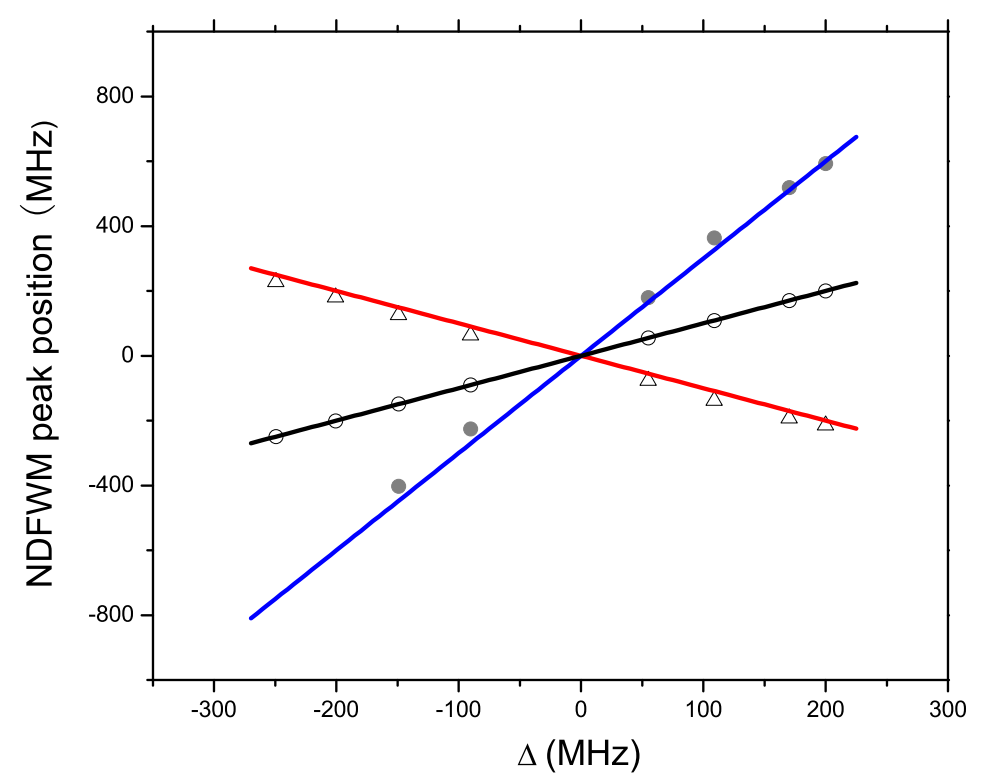




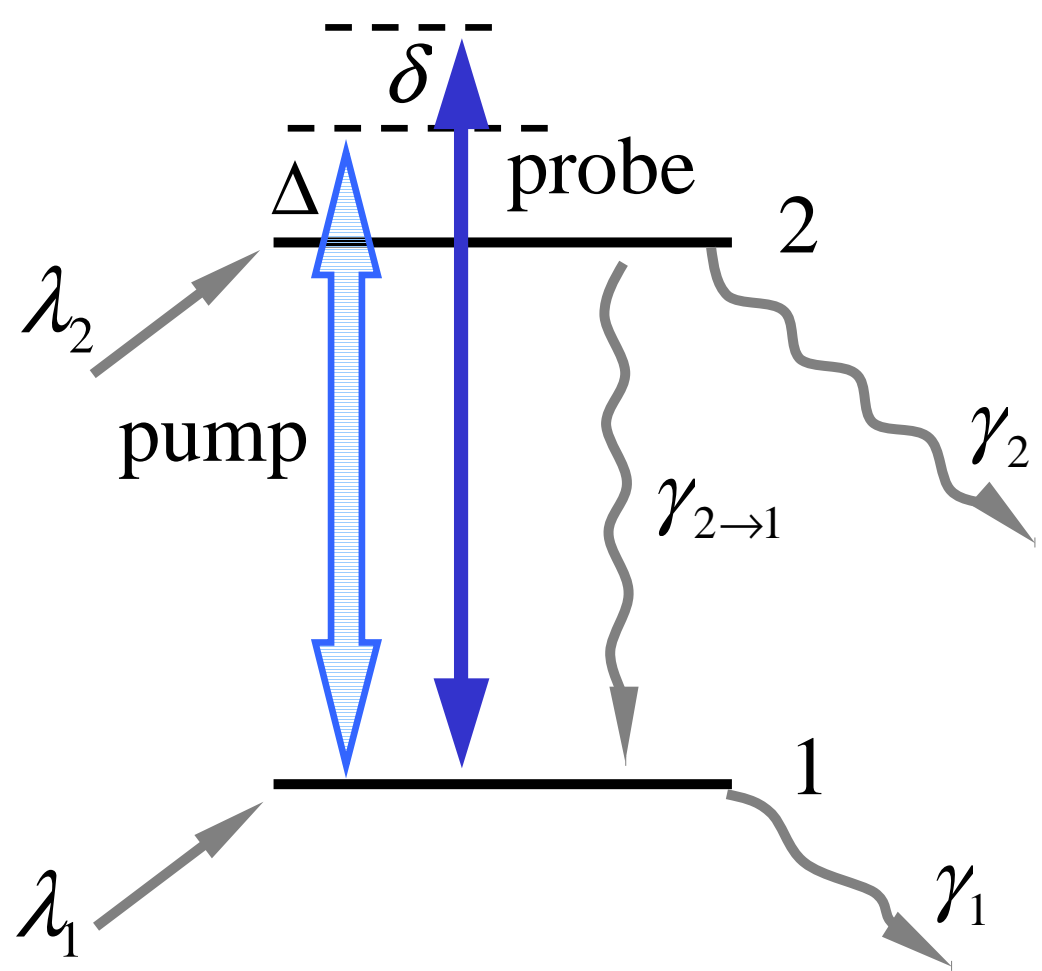

Fig. 5 

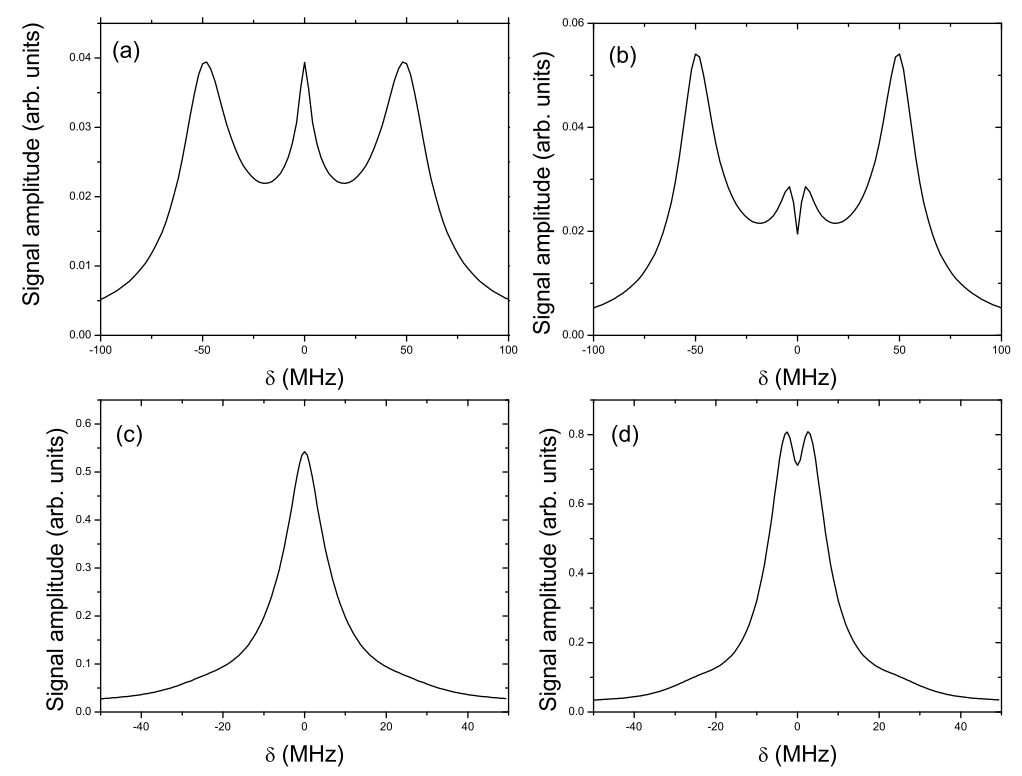